\title{
NEAR-ZERO $\Delta^{14} \mathrm{C}$ VALUES AT $32 \mathrm{KYR}$ CAL BP OBSERVED IN THE HIGH- RESOLUTION ${ }^{14} \mathrm{C}$ RECORD FROM U-Th DATED SEDIMENT OF LAKE LISAN
}

\author{
K van der Borg ${ }^{1,2} \bullet \mathrm{M} \mathrm{Stein}^{3} \bullet$ A F M de Jong ${ }^{1} \bullet \mathrm{N}$ Waldmann ${ }^{4} \bullet \mathrm{S}$ L Goldstein ${ }^{5}$
}

\begin{abstract}
A high-resolution atmospheric radiocarbon record has been obtained for the interval of 17-36 kyr from U/Thdated aragonite sediment of Lake Lisan. Reservoir age corrections were applied with reservoir ages of 200, 1250, and $2000 \mathrm{yr}$, which correlate with the different water levels of the lake. The present ${ }^{14} \mathrm{C}$ record for Lake Lisan shows near resemblance with that of Lake Suigetsu: both converge to the value of $\Delta^{14} \mathrm{C} \sim 0 \%$ at $32 \mathrm{kyr}$ cal BP. Both also show significant differences compared to other reported high-resolution ${ }^{14} \mathrm{C}$ records (e.g. Iceland Sea, Cariaco basin, and Bahamas speleothem). This inconsistency should be addressed by re-assessment of the basic assumptions behind the determination of calendar ages of the various records.
\end{abstract}

\section{INTRODUCTION}

The ratio of ${ }^{14} \mathrm{C}$ to ${ }^{12} \mathrm{C}$ in atmospheric carbon dioxide, which is the basis of radiocarbon dating, is not constant but varies due to changes in the ${ }^{14} \mathrm{C}$ production as well as changes in the carbon cycle. Thus, ${ }^{14} \mathrm{C}$ dating requires a calibration curve for transforming ${ }^{14} \mathrm{C}$ ages to calendar years. Many laboratories contributed to the present calibration curve INTCAL98 (Stuiver et al. 1998), which is a detailed record of ${ }^{14} \mathrm{C}$ age versus calendar age for the interval of 0 to $24 \mathrm{kyr}$ cal BP. The interval between 0 to $11.9 \mathrm{kyr}$ cal $\mathrm{BP}$ has been established from ${ }^{14} \mathrm{C}$ ages of tree rings identified from dendrochronology, while the portion from 11.9 to $24 \mathrm{kyr}$ cal BP relies mainly on corals and marine sediments. However, while the tree-ring archive yields a direct relation between the ${ }^{14} \mathrm{C}$ age (recorded as the atmospheric ratio of ${ }^{14} \mathrm{C}$ to ${ }^{12} \mathrm{C}$ ) versus identified calendar age, other archives are hampered either by problems with reservoir age determination or by incorrect calendar age identification (e.g. sedimentary hiatuses, problems in precise U-Th dating).

Coral samples have been used to derive calendar ages from 7-41 kyr cal BP by combining U/Th dating and accelerator mass spectrometry (AMS) ${ }^{14} \mathrm{C}$ ages (Bard et al. 1998). The atmospheric ${ }^{14} \mathrm{C} /{ }^{12} \mathrm{C}$ ratio has been deduced from the corals, assuming a constant marine reservoir age of $400 \mathrm{yr}$. However, the coral data in combination with floating chronologies yield a detailed calibration curve between 11.9 and $16 \mathrm{kyr}$ cal BP, but for the older ages, the coral data are scarce, and for the interval of 16-24 kyr cal BP, INTCAL98 uses a spline function through the coral data points.

Other detailed ${ }^{14} \mathrm{C}$ records with ages $>24 \mathrm{kyr}$ cal $\mathrm{BP}$ have been reported, but could not be used for INTCAL98 because of lacking consensus on their validity. Figure 1 shows detailed $\Delta^{14} \mathrm{C}$ records for the interval of 15-45 kyr cal BP (all with 1- $\sigma$ errors) from sediments of the Iceland Sea (Voelker et al. 1998), the Cariaco Basin (Hughen et al. 2004), Lake Suigetsu (Kitagawa and van der Plicht 1998), and a Bahamas stalagmite (Beck et al. 2001). Also shown are the coral data (Bard et al. 1998) and the prior data from Lake Lisan (Schramm et al. 2000) with corrected ${ }^{14} \mathrm{C}$ ages in HaaseSchramm et al. (2004). The various records agree reasonably well for the interval of 15-25 kyr cal BP, but for the higher ages, large discrepancies are present. The Lake Suigetsu record shows lower $\Delta \Delta^{14} \mathrm{C}$ values than the other records, whereas the stalagmite record shows higher $\Delta{ }^{14} \mathrm{C}$ values than the

\footnotetext{
${ }^{1}$ Department of Physics and Astronomy, Utrecht University, Netherlands.

${ }^{2}$ Corresponding author. Email: k.vanderborg@phys.uu.nl.

${ }^{3}$ Geological Survey of Israel, Jerusalem, Israel.

${ }^{4}$ Institute of Earth Sciences, Hebrew University, Jerusalem, Israel.

${ }^{5}$ Lamont-Doherty Earth Observatory of Columbia University, Palisades, New York 10964, USA.
} 
others. The ${ }^{14} \mathrm{C}$ records, as determined from foraminifera of the Iceland Sea and the Cariaco Basin laminae, are similar, which may be expected as both age scales, has been derived by matching the stable isotope records with the GISP2 ice core. In spite of the large differences between the records, it is remarkable that all agree with coral data. The data points for Lake Lisan (Haase-Schramm et al. 2004) also agree with the coral data and with the data for the Iceland Sea and the Cariaco Basin.

In this paper, we extend the pioneering work of Schramm et al. (2000) with a high-resolution record of Lake Lisan, the Last Glacial Dead Sea, which existed between 14-70 kyr cal BP (Kaufman 1971; Haase-Schramm et al. 2004). During its highest stand ( 170 m below mean sea level), the lake covered a large area of the Jordan-Arava Valley from the Sea of Galilee in the north to Hazeva in the south (Begin et al. 1974). The lake deposits consist of millimeter-thin laminae of aragonite and fine silty detritus, plus gypsum and thicker clastic layers. The annually-laminated authigenic aragonite recorded $\mathrm{U}$ and ${ }^{14} \mathrm{C}$ and, thus, provides an excellent opportunity to generate a high-resolution ${ }^{14} \mathrm{C}$ record for ages larger than $15 \mathrm{kyr}$ cal BP, which can be compared with the other published ${ }^{14} \mathrm{C}$ records, illuminating the way to achieve consensus among these apparently conflicting records.

\section{METHODS}

Samples were taken from the sedimentary section PZ1, located in the Perazim Valley (southwest of the present Dead Sea), which was used to determine the high-resolution U-Th chronology (HaaseSchramm et al. 2004). The lithology and geochemistry of this section has been thoroughly studied and described (Stein et al. 1997; Haase-Schramm et al. 2004). Aragonite samples were prepared from 1-2 individual laminae scratched from individual sediment blocks that were sampled continuously along the PZ1 section. The average spacing between consecutive samples is $7 \mathrm{~cm}$, corresponding to $\sim 100$ yr. Two wood pieces were found within the aragonite, allowing for direct comparison between organic and inorganic carbon.

The ${ }^{14} \mathrm{C}$ analysis was performed at Utrecht University (van der Borg et al. 1997). Acid evolution of $\mathrm{CO}_{2}$ from the carbonate samples was carried out in an evacuated glass line. Wood samples received an $\mathrm{HCl}$ wash to remove carbonate traces before they were combusted to $\mathrm{CO}_{2}$. The collected $\mathrm{CO}_{2}$ from the samples was then converted into graphite for ${ }^{14} \mathrm{C}$ analysis.

U-Th ages on the PZ1 sedimentary profile were determined by Haase-Schramm et al. (2004), who provided detailed explanation of the analytical and calculation procedures to achieve ${ }^{230} \mathrm{Th}-{ }^{234} \mathrm{U}$ of Lisan aragonite. The calendar age of the aragonite samples was obtained using the age-height regression relation $t=1.400 h+68.54$, with $h$ the height in the PZ1 section in meters and $t$ the calendar age in kyr. The $1-\sigma$ error in the calendar age was estimated at $400 \mathrm{yr}$.

\section{RESULTS AND DISCUSSION}

The measured ${ }^{14} \mathrm{C}$ ages of the aragonite samples form a record of the bi-carbonate that was provided to the lake by the incoming runoff and spring water (Stein et al. 1997). The corresponding atmospheric ${ }^{14} \mathrm{C}$ record is obtained by correcting the aragonite ages with the reservoir age of the lake at the time the aragonite was deposited. With the 2 samples of wood remains found within the aragonite layers, we assume to derive reservoir ages the corresponding ages. Table 1 shows the ${ }^{14} \mathrm{C}$ ages measured for the wood and aragonite samples. We determine the calendar age of the aragonite samples from the sample position using the above-mentioned U-Th regression. Assuming a negligible age for the wood when it became locked in the sediment, we obtain reservoir ages of $1250 \pm 180 \mathrm{yr}$ at $23.0 \mathrm{kyr}$ cal BP and $200 \pm 500 \mathrm{yr}$ at $32.7 \mathrm{kyr}$ cal BP. Previous analyses of aragoniteorganic debris pairs from the same horizon suggest a higher reservoir age of 1260 to $2000 \mathrm{yr}$ 

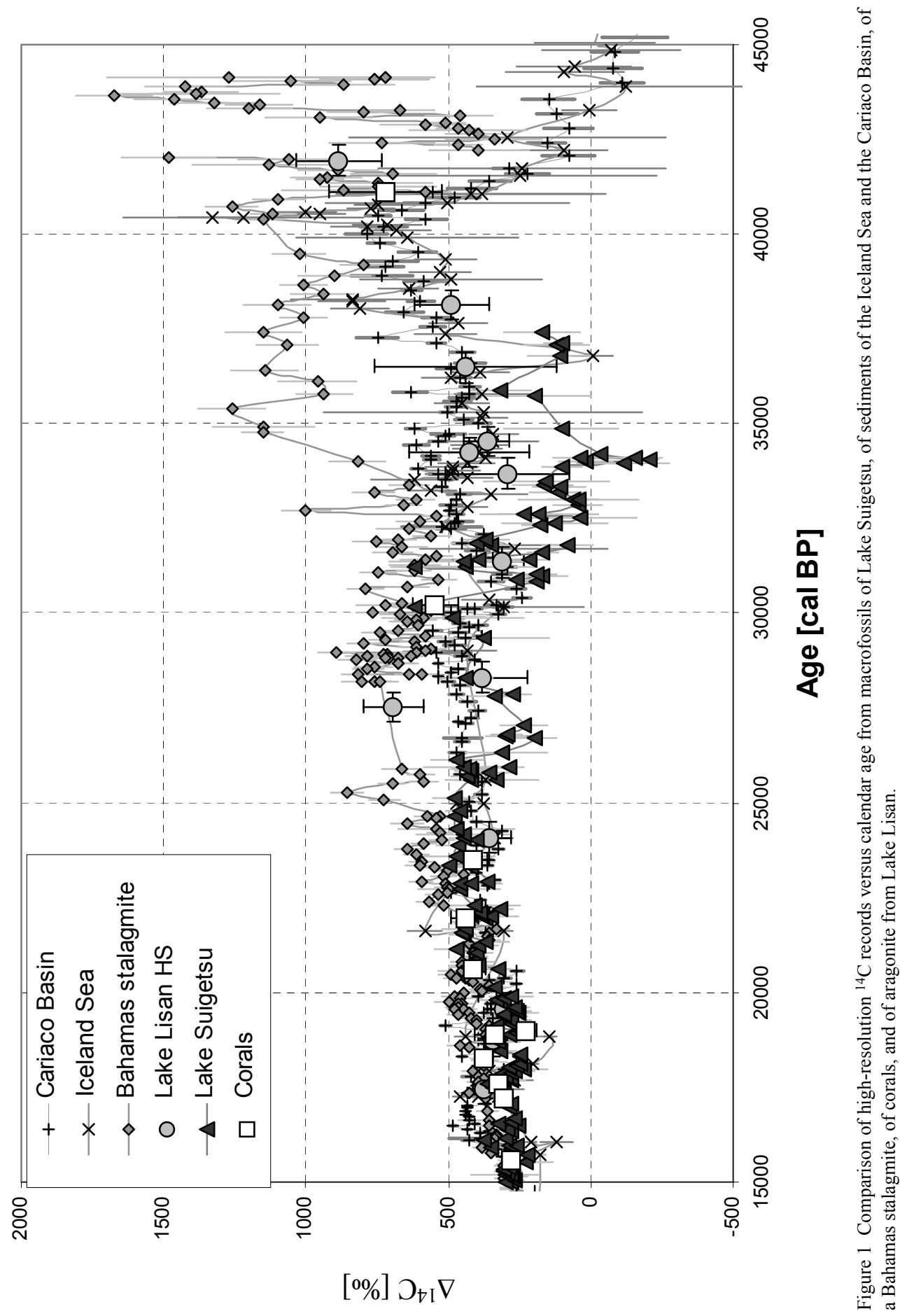
(average $=1600 \pm 250 \mathrm{yr}$ ) for the high-stand period of the lake between 19 and $26 \mathrm{kyr}$ cal BP (see data and discussion of the relation between the reservoir ages and the hydrological-limnological conditions in Stein et al., these proceedings).

Table 1 Reservoir age of Lake Lisan from ${ }^{14} \mathrm{C}$ analysis.

\begin{tabular}{llllllll}
\hline $\begin{array}{l}\text { Sample } \\
\text { name }\end{array}$ & $\begin{array}{l}\text { Height } \\
(\mathrm{cm})\end{array}$ & $\begin{array}{l}\text { U/Th age } \\
(\mathrm{cal} B \mathrm{~B})\end{array}$ & $\begin{array}{l}\text { Analyzed } \\
\text { fraction }\end{array}$ & $\begin{array}{l}\delta^{13} \mathrm{C} \\
(\%)\end{array}$ & $\begin{array}{l}{ }^{14} \mathrm{C} \text { age } \\
(\mathrm{BP})\end{array}$ & $\begin{array}{l}\text { Lab code } \\
\text { UtC- }\end{array}$ & $\begin{array}{l}\text { Reservoir age } \\
(\mathrm{BP})\end{array}$ \\
\hline ID-455w & 3247 & - & wood & -21.0 & $20,710 \pm 130$ & 12138 & - \\
ID-455a & 3247 & $23,000 \pm 400$ & aragonite & 1.6 & $21,960 \pm 130$ & 12172 & $1250 \pm 180$ \\
PZ2-2558w & 2558 & - & wood & -21.9 & $30,300 \pm 400$ & 12278 & - \\
PZ2-2558a & 2558 & $32,700 \pm 400$ & aragonite & 1.3 & $30,500 \pm 300$ & 12277 & $200 \pm 500$ \\
\hline
\end{tabular}

Using these various reservoir ages, we determine the atmospheric ${ }^{14} \mathrm{C}$ record for Lake Lisan, expressed as $\Delta{ }^{14} \mathrm{C}$ values (Table 2). The table includes the height in the section, the calendar age as calculated from the U-series age regression relationship, the estimated reservoir age, and the corresponding $\Delta^{14} \mathrm{C}$ values. The uncertainty in the calendar age from U-Th dating for the sample series is smaller than the 400-yr uncertainty deduced from the regression analysis. The average spacing for the samples in the regression analysis was $130 \mathrm{~cm}$, while the average spacing in the high-resolution record is $7 \mathrm{~cm}$. As the regression analysis assumes a constant sedimentation rate, which is likely the largest source of error, the age uncertainty for the sample series is proportional to the spacing, resulting in an average error of $20 \mathrm{yr}$.

Table 2 Results of the high-resolution ${ }^{14} \mathrm{C}$ analysis for Lake Lisan.

\begin{tabular}{|c|c|c|c|c|c|c|c|c|c|c|c|}
\hline \multirow{2}{*}{$\begin{array}{l}\begin{array}{l}\text { Sample } \\
\text { name }\end{array} \\
\text { PZ1-c14 }\end{array}$} & \multirow{2}{*}{$\begin{array}{l}\begin{array}{l}\text { Height } \\
(\mathrm{cm})\end{array} \\
3654.3\end{array}$} & \multirow{2}{*}{$\begin{array}{l}\begin{array}{l}\text { U/Th age } \\
\text { (cal BP) }\end{array} \\
17,380\end{array}$} & \multirow{2}{*}{$\begin{array}{r}\begin{array}{r}\delta^{13} \mathrm{C} \\
(\%)\end{array} \\
0.4\end{array}$} & \multicolumn{3}{|c|}{${ }^{14} \mathrm{C}$ age (BP) } & \multirow{2}{*}{$\begin{array}{l}\text { Lab code } \\
\text { UtC- } \\
11265\end{array}$} & \multirow{2}{*}{$\begin{array}{l}\text { Reservoir } \\
\text { age (BP) } \\
1250\end{array}$} & \multicolumn{3}{|c|}{$\Delta^{14} \mathrm{C}(\%)$} \\
\hline & & & & 16,240 & \pm & 80 & & & 267 & \pm & 25 \\
\hline PZ1-c13 & 3652.0 & 17,412 & 2.0 & 15,650 & \pm & 70 & 11264 & 1250 & 368 & \pm & 24 \\
\hline PZ1-c12 & 3648.6 & 17,460 & 1.2 & 15,580 & \pm & 90 & 11263 & 1250 & 388 & \pm & 31 \\
\hline PZ1-c11 & 3642.0 & 17,552 & 2.0 & 15,560 & \pm & 80 & 11262 & 1250 & 407 & \pm & 28 \\
\hline PZ1-c10 & 3639.2 & 17,591 & 3.2 & 15,720 & \pm & 80 & 11261 & 1250 & 386 & \pm & 28 \\
\hline PZ1-c9 & 3632.5 & 17,685 & 1.1 & 15,890 & \pm & 80 & 11260 & 1250 & 373 & \pm & 27 \\
\hline PZ1-c8 & 3626.4 & 17,770 & 2.3 & 16,250 & \pm & 80 & 11259 & 1250 & 326 & \pm & 26 \\
\hline PZ1-c7 & 3620.2 & 17,857 & 1.0 & 16,380 & \pm & 90 & 11245 & 1250 & 319 & \pm & 30 \\
\hline PZ1-c6 & 3613.8 & 17,947 & 2.6 & 16,410 & \pm & 100 & 11244 & 1250 & 328 & \pm & 33 \\
\hline PZ1-c5 & 3610.0 & 18,000 & 3.3 & 16,330 & \pm & 90 & 11243 & 1250 & 350 & \pm & 30 \\
\hline PZ1-c4 & 3609.5 & 18,007 & 3.2 & 16,260 & \pm & 90 & 11242 & 1250 & 363 & \pm & 31 \\
\hline PZ1-c3 & 3608.8 & 18,017 & 1.7 & 16,220 & \pm & 100 & 11241 & 1250 & 371 & \pm & 34 \\
\hline PZ1-c2 & 3608.2 & 18,025 & 0.7 & 15,620 & \pm & 90 & 11240 & 1250 & 479 & \pm & 33 \\
\hline PZ1-c1 & 3607.5 & 18,035 & 1.1 & 15,910 & \pm & 100 & 11239 & 1250 & 428 & \pm & 36 \\
\hline PZ1-c15 & 3603.8 & 18,087 & 2.1 & 16,460 & \pm & 80 & 11266 & 1250 & 342 & \pm & 27 \\
\hline PZ1-c16 & 3598.5 & 18,161 & 0.6 & 16,240 & \pm & 80 & 11267 & 1250 & 392 & \pm & 28 \\
\hline PZ1-c17 & 3593.5 & 18,231 & 3.0 & 16,380 & \pm & 80 & 11268 & 1250 & 380 & \pm & 27 \\
\hline PZ1-c18 & 3591.8 & 18,255 & 3.3 & 16,420 & \pm & 90 & 11246 & 1250 & 377 & \pm & 31 \\
\hline PZ1-c19 & 3584.8 & 18,353 & 3.3 & 16,420 & \pm & 90 & 11269 & 1250 & 393 & \pm & 31 \\
\hline PZ1-c20 & 3579.7 & 18,424 & 1.7 & 16,520 & \pm & 80 & 11270 & 1250 & 388 & \pm & 28 \\
\hline PZ1-c21 & 3575.2 & 18,487 & 1.6 & 16,870 & \pm & 80 & 11271 & 1250 & 339 & \pm & 27 \\
\hline PZ1-c22 & 3567.5 & 18,595 & 0.7 & 16,890 & \pm & 90 & 11272 & 1250 & 353 & \pm & 30 \\
\hline PZ1-c23 & 3562.6 & 18,664 & -0.5 & 16,890 & \pm & 100 & 11273 & 1250 & 364 & \pm & 34 \\
\hline PZ1-c24 & 3557.5 & 18,735 & 0.1 & 16,950 & \pm & 100 & 11274 & 1250 & 366 & \pm & 34 \\
\hline PZ1-c25 & 3552.2 & 18,809 & 1.8 & 16,990 & \pm & 90 & 11275 & 1250 & 371 & \pm & 31 \\
\hline PZ1-c26 & 3545.6 & 18,902 & 0.2 & 17,050 & \pm & 100 & 11276 & 1250 & 376 & \pm & 34 \\
\hline PZ1-c27 & 3539.4 & 18,988 & 2.7 & 17,200 & \pm & 90 & 11277 & 1250 & 365 & \pm & 31 \\
\hline PZ1-c28 & 3533.0 & 19,078 & 3.2 & 17,630 & \pm & 100 & 11247 & 1250 & 308 & \pm & 33 \\
\hline
\end{tabular}


Table 2 Results of the high-resolution ${ }^{14} \mathrm{C}$ analysis for Lake Lisan. (Continued)

\begin{tabular}{|c|c|c|c|c|c|c|c|c|c|c|c|}
\hline $\begin{array}{l}\text { Sample } \\
\text { name }\end{array}$ & $\begin{array}{l}\text { Height } \\
(\mathrm{cm})\end{array}$ & $\begin{array}{l}\text { U/Th age } \\
\text { (cal BP) }\end{array}$ & $\begin{array}{l}\delta^{13} \mathrm{C} \\
(\%)\end{array}$ & \multicolumn{3}{|c|}{${ }^{14} \mathrm{C}$ age (BP) } & $\begin{array}{l}\text { Lab code } \\
\text { UtC- }\end{array}$ & $\begin{array}{l}\text { Reservoir } \\
\text { age (BP) }\end{array}$ & \multicolumn{3}{|c|}{$\Delta^{14} \mathrm{C}(\%)$} \\
\hline PZ1-c29 & 3528.8 & 19,137 & 0.5 & 17,500 & \pm & 90 & 11278 & 1250 & 339 & \pm & 30 \\
\hline PZ1-c30 & 3522.3 & 19,228 & 1.1 & 17,440 & \pm & 100 & 11279 & 1250 & 364 & \pm & 34 \\
\hline PZ1-c31 & 3516.8 & 19,305 & 3.5 & 17,960 & \pm & 100 & 11280 & 1250 & 290 & \pm & 32 \\
\hline PZ1-c32 & 3510.9 & 19,387 & 0.7 & 18,070 & \pm & 110 & 11281 & 1250 & 286 & \pm & 35 \\
\hline PZ1-c33 & 3506.6 & 19,448 & 0.2 & 18,010 & \pm & 100 & 11282 & 1250 & 305 & \pm & 32 \\
\hline PZ1-c34 & 3501.0 & 19,526 & 1.3 & 18,426 & \pm & 100 & 11283 & 1250 & 251 & \pm & 31 \\
\hline PZ1-c35 & 3495.1 & 19,609 & 2.1 & 18,280 & \pm & 100 & 11284 & 1250 & 286 & \pm & 32 \\
\hline PZ1-c36 & 3489.4 & 19,688 & 1.0 & 18,100 & \pm & 100 & 11285 & 1250 & 328 & \pm & 33 \\
\hline PZ1-c37 & 3483.7 & 19,768 & 2.4 & 18,290 & \pm & 100 & 11286 & 1250 & 310 & \pm & 33 \\
\hline PZ1-c38 & 3477.9 & 19,849 & 1.7 & 18,510 & \pm & 110 & 11248 & 1250 & 287 & \pm & 35 \\
\hline PZ1-c39 & 3472.2 & 19,929 & 1.7 & 19,070 & \pm & 100 & 11287 & 1250 & 212 & \pm & 30 \\
\hline PZ1-c40 & 3467.2 & 19,999 & 0.6 & 18,280 & \pm & 110 & 11288 & 1250 & 349 & \pm & 37 \\
\hline PZ1-c41 & 3462.2 & 20,069 & 1.5 & 18,690 & \pm & 110 & 11289 & 1250 & 293 & \pm & 35 \\
\hline PZ1-c42 & 3455.4 & 20,164 & 5.7 & 18,990 & \pm & 130 & 11290 & 1250 & 260 & \pm & 41 \\
\hline PZ1-c43 & 3443.3 & 20,334 & 1.3 & 19,150 & \pm & 130 & 11291 & 1250 & 260 & \pm & 41 \\
\hline PZ1-c44 & 3438.3 & 20,404 & 0.4 & 18,740 & \pm & 110 & 11292 & 1250 & 338 & \pm & 37 \\
\hline PZ1-c45 & 3432.1 & 20,491 & 1.2 & 18,950 & \pm & 120 & 11293 & 1250 & 317 & \pm & 39 \\
\hline PZ1-c46 & 3426.4 & 20,570 & 2.2 & 19,120 & \pm & 110 & 11294 & 1250 & 302 & \pm & 36 \\
\hline PZ1-c47 & 3420.0 & 20,660 & 3.9 & 18,910 & \pm & 130 & 11295 & 1250 & 351 & \pm & 44 \\
\hline PZ1-c48 & 3414.4 & 20,738 & 3.3 & 19,270 & \pm & 120 & 11249 & 1250 & 304 & \pm & 39 \\
\hline PZ1-c49 & 3406.3 & 20,852 & 1.9 & 19,220 & \pm & 120 & 11296 & 2000 & 460 & \pm & 44 \\
\hline PZ1-c50 & 3398.7 & 20,958 & 0.8 & 19,220 & \pm & 120 & 11297 & 2000 & 479 & \pm & 44 \\
\hline PZ1-c51 & 3395.3 & 21,006 & 1.4 & 19,820 & \pm & 170 & 11298 & 2000 & 381 & \pm & 58 \\
\hline PZ1-c52 & 3392.4 & 21,046 & 1.8 & 19,330 & \pm & 130 & 11299 & 2000 & 475 & \pm & 48 \\
\hline PZ1-c53 & 3387.6 & 21,114 & 2.4 & 19,620 & \pm & 120 & 11300 & 2000 & 434 & \pm & 43 \\
\hline PZ1-c54 & 3379.1 & 21,233 & 1.9 & 19,660 & \pm & 130 & 11301 & 2000 & 448 & \pm & 47 \\
\hline PZ1-c55 & 3373.4 & 21,312 & -0.4 & 19,520 & \pm & 110 & 11302 & 2000 & 487 & \pm & 41 \\
\hline PZ1-c56 & 3368.2 & 21,385 & 2.9 & 19,810 & \pm & 110 & 11303 & 2000 & 447 & \pm & 40 \\
\hline PZ1-c57 & 3363.0 & 21,458 & 0.3 & 19,860 & \pm & 120 & 11304 & 2000 & 451 & \pm & 43 \\
\hline PZ1-c58 & 3357.4 & 21,536 & 0.5 & 19,720 & \pm & 130 & 11250 & 2000 & 491 & \pm & 48 \\
\hline PZ1-c59 & 3354.5 & 21,577 & 0.9 & 19,520 & \pm & 120 & 11305 & 2000 & 536 & \pm & 46 \\
\hline PZ1-c60 & 3350.8 & 21,629 & 2.9 & 19,840 & \pm & 120 & 11306 & 2000 & 485 & \pm & 44 \\
\hline PZ1-c61 & 3346.0 & 21,696 & 4.3 & 19,910 & \pm & 130 & 11307 & 2000 & 484 & \pm & 48 \\
\hline PZ1-c62 & 3342.0 & 21,752 & 0.5 & 20,030 & \pm & 130 & 11308 & 2000 & 472 & \pm & 48 \\
\hline PZ1-c63 & 3336.6 & 21,828 & 3.1 & 20,160 & \pm & 120 & 11309 & 2000 & 462 & \pm & 44 \\
\hline PZ1-c64 & 3331.4 & 21,900 & 3.4 & 20,140 & \pm & 130 & 11310 & 2000 & 478 & \pm & 48 \\
\hline PZ1-c65 & 3327.3 & 21,958 & 0.8 & 20,340 & \pm & 120 & 11311 & 2000 & 452 & \pm & 43 \\
\hline PZ1-c66 & 3321.2 & 22,043 & 2.5 & 20,470 & \pm & 120 & 11312 & 2000 & 444 & \pm & 43 \\
\hline PZ1-c67 & 3312.6 & 22,164 & 2.9 & 20,820 & \pm & 140 & 11313 & 2000 & 402 & \pm & 49 \\
\hline PZ1-c68 & 3307.0 & 22,242 & 1.3 & 20,810 & \pm & 140 & 11251 & 2000 & 417 & \pm & 49 \\
\hline PZ1-c69 & 3300.4 & 22,334 & 0.8 & 20,640 & \pm & 140 & 11314 & 2000 & 464 & \pm & 51 \\
\hline PZ1-c70 & 3292.2 & 22,449 & -0.3 & 20,890 & \pm & 140 & 11315 & 2000 & 439 & \pm & 50 \\
\hline PZ1-c71 & 3286.0 & 22,536 & 0.0 & 21,640 & \pm & 130 & 11316 & 2000 & 325 & \pm & 43 \\
\hline PZ1-c72 & 3280.7 & 22,610 & 0.4 & 21,070 & \pm & 130 & 11317 & 2000 & 435 & \pm & 46 \\
\hline PZ1-c73 & 3275.5 & 22,683 & -0.3 & 21,230 & \pm & 140 & 11318 & 2000 & 419 & \pm & 49 \\
\hline PZ1-c74 & 3270.2 & 22,757 & 0.3 & 21,470 & \pm & 130 & 11319 & 2000 & 390 & \pm & 45 \\
\hline PZ1-c75 & 3265.6 & 22,822 & 1.8 & 21,080 & \pm & 120 & 11320 & 2000 & 470 & \pm & 44 \\
\hline PZ1-c76 & 3260.4 & 22,894 & 0.4 & 21,210 & \pm & 140 & 11321 & 2000 & 459 & \pm & 51 \\
\hline PZ1-c77 & 3256.8 & 22,945 & 2.0 & 21,230 & \pm & 140 & 11322 & 2000 & 465 & \pm & 51 \\
\hline PZ1-c78 & 3249.1 & 23,053 & 2.8 & 21,480 & \pm & 170 & 11252 & 2000 & 438 & \pm & 61 \\
\hline PZ1-c79 & 3244.4 & 23,118 & -0.7 & 22,110 & \pm & 150 & 11323 & 2000 & 341 & \pm & 50 \\
\hline PZ1-c80 & 3239.0 & 23,194 & 0.2 & 22,350 & \pm & 140 & 11324 & 2000 & 313 & \pm & 46 \\
\hline PZ1-c81 & 3232.2 & 23,289 & 0.5 & 21,710 & \pm & 160 & 11325 & 2000 & 438 & \pm & 57 \\
\hline PZ1-c82 & 3226.0 & 23,376 & 0.1 & 21,950 & \pm & 140 & 11326 & 2000 & 411 & \pm & 49 \\
\hline PZ1-c83 & 3218.1 & 23,487 & 0.1 & 22,010 & \pm & 140 & 11327 & 2000 & 419 & \pm & 49 \\
\hline
\end{tabular}


Table 2 Results of the high-resolution ${ }^{14} \mathrm{C}$ analysis for Lake Lisan. (Continued)

\begin{tabular}{|c|c|c|c|c|c|c|c|c|c|c|c|}
\hline $\begin{array}{l}\text { Sample } \\
\text { name }\end{array}$ & $\begin{array}{l}\text { Height } \\
(\mathrm{cm})\end{array}$ & $\begin{array}{l}\text { U/Th age } \\
\text { (cal BP) }\end{array}$ & $\begin{array}{l}\delta^{13} \mathrm{C} \\
(\%)\end{array}$ & \multicolumn{3}{|c|}{${ }^{14} \mathrm{C}$ age (BP) } & $\begin{array}{l}\text { Lab code } \\
\text { UtC- }\end{array}$ & $\begin{array}{l}\text { Reservoir } \\
\text { age (BP) }\end{array}$ & \multicolumn{3}{|c|}{$\Delta^{14} \mathrm{C}(\%)$} \\
\hline PZ1-c84 & 3204.7 & 23,674 & 1.6 & 21,850 & \pm & 140 & 11328 & 2000 & 481 & \pm & 52 \\
\hline PZ1-c85 & 3196.4 & 23,790 & 0.2 & 22,230 & \pm & 140 & 11329 & 2000 & 432 & \pm & 50 \\
\hline PZ1-c86 & 3188.4 & 23,902 & 1.3 & 22,310 & \pm & 140 & 11330 & 2000 & 438 & \pm & 50 \\
\hline PZ1-c87 & 3181.6 & 23,998 & 1.6 & 22,310 & \pm & 160 & 11331 & 2000 & 454 & \pm & 58 \\
\hline PZ1-c88 & 3174.2 & 24,101 & 1.1 & 22,450 & \pm & 170 & 11253 & 2000 & 447 & \pm & 61 \\
\hline PZ1-c89 & 3167.8 & 24,191 & 1.4 & 22,680 & \pm & 150 & 11332 & 2000 & 422 & \pm & 53 \\
\hline PZ1-c90 & 3157.9 & 24,329 & 1.3 & 22,680 & \pm & 150 & 11333 & 2000 & 446 & \pm & 54 \\
\hline PZ1-c91 & 3149.0 & 24,454 & 1.8 & 22,680 & \pm & 160 & 11334 & 2000 & 468 & \pm & 58 \\
\hline PZ1-c92 & 3141.2 & 24,563 & 1.5 & 22,640 & \pm & 150 & 11335 & 2000 & 495 & \pm & 56 \\
\hline PZ1-c93 & 3133.1 & 24,677 & 1.8 & 23,070 & \pm & 140 & 11336 & 2000 & 436 & \pm & 50 \\
\hline PZ1-c94 & 3125.7 & 24,780 & -0.5 & 23,390 & \pm & 180 & 11254 & 2000 & 398 & \pm & 63 \\
\hline PZ1-c97 & 3102.4 & 25,106 & 1.5 & 23,420 & \pm & 100 & 11622 & 2000 & 448 & \pm & 36 \\
\hline PZ1-c98 & 3095.4 & 25,204 & 1.4 & 23,370 & \pm & 120 & 11623 & 1250 & 343 & \pm & 40 \\
\hline PZ1-c99 & 3084.3 & 25,360 & 1.0 & 23,200 & \pm & 100 & 11624 & 1250 & 398 & \pm & 35 \\
\hline PZ1-c100 & 3074.4 & 25,498 & -0.2 & 23,480 & \pm & 110 & 11625 & 1250 & 373 & \pm & 38 \\
\hline PZ1-c101 & 3063.5 & 25,651 & 2.0 & 23,700 & \pm & 120 & 11626 & 1250 & 361 & \pm & 41 \\
\hline PZ1-c102 & 3053.5 & 25,791 & 1.3 & 23,640 & \pm & 120 & 11627 & 1250 & 394 & \pm & 42 \\
\hline PZ1-c103 & 3046.3 & 25,892 & 0.8 & 23,600 & \pm & 120 & 11628 & 1250 & 419 & \pm & 42 \\
\hline PZ1-c104 & 3038.7 & 25,998 & 1.5 & 23,940 & \pm & 110 & 11629 & 1250 & 377 & \pm & 38 \\
\hline PZ1-c105 & 3029.7 & 26,124 & 0.9 & 24,380 & \pm & 140 & 11630 & 1250 & 324 & \pm & 46 \\
\hline PZ1-c106 & 3020.2 & 26,257 & 2.1 & 24,070 & \pm & 110 & 11631 & 1250 & 398 & \pm & 38 \\
\hline PZ1-c107 & 3010.9 & 26,387 & 0.1 & 24,280 & \pm & 120 & 11632 & 1250 & 384 & \pm & 41 \\
\hline PZ1-c108 & 2999.7 & 26,544 & 1.3 & 24,630 & \pm & 120 & 11633 & 1250 & 350 & \pm & 40 \\
\hline PZ1-c109 & 2991.0 & 26,666 & 2.6 & 24,630 & \pm & 120 & 11634 & 1250 & 370 & \pm & 41 \\
\hline PZ1-c110 & 2982.4 & 26,786 & 2.4 & 25,040 & \pm & 110 & 11635 & 1250 & 321 & \pm & 36 \\
\hline PZ1-c111 & 2972.9 & 26,919 & 2.2 & 25,000 & \pm & 110 & 11636 & 1250 & 349 & \pm & 37 \\
\hline PZ1-c112 & 2965.3 & 27,026 & 0.2 & 25,110 & \pm & 110 & 11637 & 1250 & 348 & \pm & 37 \\
\hline PZ1-c113 & 2959.0 & 27,114 & 0.7 & 25,390 & \pm & 110 & 11638 & 1250 & 316 & \pm & 36 \\
\hline PZ1-c114 & 2953.3 & 27,194 & 1.7 & 25,220 & \pm & 130 & 11639 & 1250 & 357 & \pm & 44 \\
\hline PZ1-c115 & 2947.5 & 27,275 & 2.0 & 25,240 & \pm & 130 & 11640 & 1250 & 367 & \pm & 44 \\
\hline PZ1-c116 & 2940.3 & 27,376 & 1.4 & 25,470 & \pm & 120 & 11641 & 1250 & 345 & \pm & 40 \\
\hline PZ1-c117 & 2933.0 & 27,478 & 3.0 & 25,480 & \pm & 120 & 11642 & 1250 & 360 & \pm & 41 \\
\hline PZ1-c118 & 2927.2 & 27,559 & -0.5 & 26,160 & \pm & 130 & 11643 & 1250 & 262 & \pm & 41 \\
\hline PZ1-c119 & 2923.1 & 27,617 & 0.3 & 26,210 & \pm & 130 & 11644 & 1250 & 263 & \pm & 41 \\
\hline PZ1-c122 & 2907.5 & 27,835 & 1.8 & 25,740 & \pm & 120 & 11647 & 1250 & 375 & \pm & 41 \\
\hline PZ1-c120 & 2906.4 & 27,850 & 1.5 & 26,080 & \pm & 130 & 11645 & 1250 & 320 & \pm & 43 \\
\hline PZ1-c121 & 2899.7 & 27,944 & 0.9 & 25,840 & \pm & 140 & 11646 & 1250 & 376 & \pm & 48 \\
\hline PZ1-c123 & 2893.8 & 28,027 & 1.6 & 26,290 & \pm & 130 & 11648 & 1250 & 314 & \pm & 43 \\
\hline PZ1-c124 & 2884.8 & 28,153 & 0.7 & 26,250 & \pm & 130 & 11649 & 1250 & 341 & \pm & 43 \\
\hline PZ1-c125 & 2876.6 & 28,268 & 1.9 & 26,380 & \pm & 150 & 11650 & 1250 & 338 & \pm & 50 \\
\hline PZ1-c126 & 2868.4 & 28,382 & 1.3 & 26,590 & \pm & 130 & 11651 & 1250 & 321 & \pm & 43 \\
\hline PZ1-c127 & 2863.0 & 28,458 & 1.7 & 26,930 & \pm & 120 & 11652 & 1250 & 278 & \pm & 38 \\
\hline PZ1-c128 & 2855.4 & 28,564 & 1.5 & 26,860 & \pm & 140 & 11653 & 1250 & 306 & \pm & 46 \\
\hline PZ1-c129 & 2852.4 & 28,606 & 1.2 & 27,210 & \pm & 140 & 11654 & 1250 & 257 & \pm & 44 \\
\hline PZ1-c130 & 2841.9 & 28,753 & 1.9 & 26,860 & \pm & 120 & 11655 & 1250 & 336 & \pm & 40 \\
\hline PZ1-c131 & 2835.2 & 28,847 & 1.4 & 26,880 & \pm & 120 & 11656 & 1250 & 348 & \pm & 40 \\
\hline PZ1-c132 & 2832.2 & 28,889 & 2.0 & 27,120 & \pm & 120 & 11657 & 1250 & 315 & \pm & 39 \\
\hline PZ1-c133 & 2828.6 & 28,940 & 1.5 & 27,230 & \pm & 120 & 11658 & 1250 & 305 & \pm & 39 \\
\hline PZ1-c134 & 2826.7 & 28,966 & 2.0 & 27,160 & \pm & 140 & 11659 & 1250 & 321 & \pm & 46 \\
\hline PZ1-c135 & 2820.5 & 29,053 & 1.3 & 27,580 & \pm & 150 & 11660 & 1250 & 267 & \pm & 47 \\
\hline PZ1-c136 & 2818.8 & 29,077 & 1.0 & 27,650 & \pm & 150 & 11661 & 1250 & 260 & \pm & 47 \\
\hline PZ1-c137 & 2813.4 & 29,152 & 0.5 & 27,700 & \pm & 130 & 11662 & 1250 & 263 & \pm & 41 \\
\hline PZ1-c138 & 2804.8 & 29,273 & 0.4 & 27,780 & \pm & 130 & 11663 & 1250 & 269 & \pm & 41 \\
\hline PZ1-c139 & 2796.1 & 29,395 & 1.6 & 27,550 & \pm & 150 & 11664 & 1250 & 325 & \pm & 49 \\
\hline PZ1-c140 & 2789.4 & 29,488 & 3.3 & 27,600 & \pm & 150 & 11665 & 1250 & 332 & \pm & 50 \\
\hline
\end{tabular}


Table 2 Results of the high-resolution ${ }^{14} \mathrm{C}$ analysis for Lake Lisan. (Continued)

\begin{tabular}{|c|c|c|c|c|c|c|c|c|c|c|c|}
\hline $\begin{array}{l}\text { Sample } \\
\text { name }\end{array}$ & $\begin{array}{l}\text { Height } \\
(\mathrm{cm})\end{array}$ & $\begin{array}{l}\text { U/Th age } \\
\text { (cal BP) }\end{array}$ & $\begin{array}{l}\delta^{13} \mathrm{C} \\
(\%)\end{array}$ & \multicolumn{3}{|c|}{${ }^{14} \mathrm{C}$ age (BP) } & $\begin{array}{l}\text { Lab code } \\
\text { UtC- }\end{array}$ & $\begin{array}{l}\text { Reservoir } \\
\text { age (BP) }\end{array}$ & \multicolumn{3}{|c|}{$\Delta^{14} \mathrm{C}(\%)$} \\
\hline PZ1-c141 & 2782.4 & 29,586 & 1.4 & 28,040 & \pm & 160 & 11666 & 1250 & 276 & \pm & 51 \\
\hline PZ1-c142 & 2775.0 & 29,690 & 1.7 & 27,430 & \pm & 120 & 11692 & 1250 & 394 & \pm & 42 \\
\hline PZ1-c143 & 2767.4 & 29,796 & 1.4 & 28,410 & \pm & 140 & 11693 & 1250 & 250 & \pm & 44 \\
\hline PZ1-c144 & 2759.3 & 29,910 & 1.1 & 28,720 & \pm & 150 & 11694 & 1250 & 219 & \pm & 46 \\
\hline PZ1-c145 & 2752.1 & 30,011 & 1.5 & 28,440 & \pm & 140 & 11695 & 1250 & 278 & \pm & 45 \\
\hline PZ1-c146 & 2743.8 & 30,127 & 1.4 & 28,750 & \pm & 150 & 11696 & 1250 & 247 & \pm & 47 \\
\hline PZ1-c147 & 2736.8 & 30,225 & 2.0 & 28,750 & \pm & 150 & 11697 & 1250 & 262 & \pm & 47 \\
\hline PZ1-c148 & 2730.1 & 30,319 & 1.8 & 28,990 & \pm & 150 & 11698 & 1250 & 239 & \pm & 46 \\
\hline PZ1-c149 & 2724.1 & 30,403 & 3.6 & 29,130 & \pm & 150 & 11699 & 1250 & 230 & \pm & 46 \\
\hline PZ1-c150 & 2714.6 & 30,536 & 2.3 & 29,260 & \pm & 190 & 11700 & 1250 & 230 & \pm & 58 \\
\hline PZ1-c151 & 2705.8 & 30,659 & 0.5 & 29,260 & \pm & 160 & 11701 & 1250 & 248 & \pm & 50 \\
\hline PZ1-c152 & 2699.0 & 30,754 & 2.1 & 29,230 & \pm & 150 & 11702 & 1250 & 267 & \pm & 47 \\
\hline PZ1-c153 & 2690.1 & 30,879 & 1.5 & 29,410 & \pm & 190 & 11703 & 1250 & 258 & \pm & 60 \\
\hline PZ1-c154 & 2687.0 & 30,922 & 1.5 & 29,130 & \pm & 150 & 11704 & 1250 & 310 & \pm & 49 \\
\hline PZ1-c155 & 2680.1 & 31,019 & 1.7 & 29,130 & \pm & 150 & 11705 & 1250 & 325 & \pm & 49 \\
\hline PZ1-c156 & 2672.2 & 31,129 & 2.0 & 29,630 & \pm & 160 & 11706 & 1250 & 262 & \pm & 50 \\
\hline PZ1-c157 & 2667.0 & 31,202 & 1.8 & 29,470 & \pm & 160 & 11707 & 1250 & 299 & \pm & 52 \\
\hline PZ1-c158 & 2661.7 & 31,276 & 1.2 & 30,030 & \pm & 170 & 11708 & 1250 & 222 & \pm & 52 \\
\hline PZ1-c159 & 2638.7 & 31,598 & 0.5 & 29,800 & \pm & 300 & 12380 & 1250 & 308 & \pm & 98 \\
\hline PZ1-c160 & 2637.7 & 31,612 & -0.1 & 29,900 & \pm & 300 & 12381 & 1250 & 294 & \pm & 97 \\
\hline PZ1-c161 & 2629.7 & 31,724 & -3.9 & 28,800 & \pm & 300 & 12382 & 1250 & 504 & \pm & 112 \\
\hline PZ1-c162 & 2621.9 & 31,833 & -0.1 & 29,700 & \pm & 300 & 12383 & 1250 & 362 & \pm & 102 \\
\hline PZ1-c163 & 2616.1 & 31,915 & 1.0 & 30,200 & \pm & 300 & 12384 & 1250 & 293 & \pm & 97 \\
\hline PZ1-c164 & 2613.8 & 31,947 & 0.5 & 29,600 & \pm & 300 & 12385 & 1250 & 398 & \pm & 104 \\
\hline PZ1-c165 & 2608.0 & 32,028 & 2.0 & 30,700 & \pm & 280 & 12386 & 1250 & 231 & \pm & 86 \\
\hline PZ1-c166 & 2600.5 & 32,133 & 1.9 & 30,600 & \pm & 280 & 12387 & 200 & 108 & \pm & 77 \\
\hline PZ1-c167 & 2594.6 & 32,216 & -1.4 & 31,100 & \pm & 350 & 12388 & 200 & 52 & \pm & 92 \\
\hline PZ1-c168 & 2588.2 & 32,305 & -1.1 & 30,600 & \pm & 290 & 12389 & 200 & 131 & \pm & 82 \\
\hline PZ1-c169 & 2586.7 & 32,326 & 2.8 & 30,700 & \pm & 300 & 12390 & 200 & 120 & \pm & 84 \\
\hline PZ1-c170 & 2586.2 & 32,333 & 1.0 & 31,300 & \pm & 300 & 12391 & 200 & 40 & \pm & 78 \\
\hline PZ1-c171 & 2582.8 & 32,381 & -0.5 & 31,600 & \pm & 300 & 12392 & 200 & 8 & \pm & 75 \\
\hline PZ1-c172 & 2570.4 & 32,554 & -3.3 & 31,500 & \pm & 350 & 12393 & 200 & 42 & \pm & 91 \\
\hline PZ1-c173 & 2567.6 & 32,594 & -1.1 & 32,000 & \pm & 350 & 12394 & 200 & -16 & \pm & 86 \\
\hline PZ1-c174 & 2547.7 & 32,872 & -0.8 & 31,200 & \pm & 350 & 12395 & 200 & 124 & \pm & 98 \\
\hline PZ1-c175 & 2544.3 & 32,920 & -0.5 & 31,600 & \pm & 300 & 12396 & 200 & 76 & \pm & 80 \\
\hline PZ1-c176 & 2537.6 & 33,014 & -0.7 & 31,700 & \pm & 300 & 12397 & 200 & 75 & \pm & 80 \\
\hline PZ1-c177 & 2530.1 & 33,119 & 0.9 & 31,100 & \pm & 300 & 12398 & 200 & 173 & \pm & 88 \\
\hline PZ1-c178 & 2524.8 & 33,193 & 1.6 & 31,500 & \pm & 300 & 12399 & 200 & 126 & \pm & 84 \\
\hline PZ1-c179 & 2519.0 & 33,274 & 2.4 & 31,500 & \pm & 300 & 12400 & 200 & 137 & \pm & 85 \\
\hline PZ1-c180 & 2514.1 & 33,343 & 0.4 & 31,700 & \pm & 300 & 12401 & 200 & 118 & \pm & 84 \\
\hline PZ1-c181 & 2508.1 & 33,427 & 0.6 & 31,900 & \pm & 350 & 12402 & 200 & 102 & \pm & 96 \\
\hline PZ1-c182 & 2502.1 & 33,511 & 1.7 & 32,100 & \pm & 350 & 12403 & 200 & 86 & \pm & 95 \\
\hline PZ1-c183 & 2497.0 & 33,582 & 1.8 & 31,900 & \pm & 350 & 12404 & 200 & 123 & \pm & 98 \\
\hline PZ1-c184 & 2491.0 & 33,666 & 1.4 & 32,400 & \pm & 350 & 12405 & 200 & 66 & \pm & 93 \\
\hline PZ1-c185 & 2484.8 & 33,753 & 1.0 & 32,000 & \pm & 350 & 12406 & 200 & 132 & \pm & 99 \\
\hline PZ1-c186 & 2477.1 & 33,861 & 0.7 & 32,100 & \pm & 400 & 12407 & 200 & 133 & \pm & 113 \\
\hline PZ1-c187 & 2470.9 & 33,947 & 1.0 & 32,200 & \pm & 400 & 12408 & 200 & 131 & \pm & 113 \\
\hline PZ1-c188 & 2463.6 & 34,050 & 0.7 & 32,400 & \pm & 400 & 12409 & 200 & 117 & \pm & 111 \\
\hline PZ1-c189 & 2455.3 & 34,166 & 0.6 & 32,300 & \pm & 400 & 12410 & 200 & 147 & \pm & 114 \\
\hline PZ1-c190 & 2454.3 & 34,180 & -2.2 & 32,100 & \pm & 350 & 12411 & 200 & 177 & \pm & 103 \\
\hline PZ1-c191 & 2447.9 & 34,269 & 1.0 & 32,300 & \pm & 400 & 12412 & 200 & 161 & \pm & 116 \\
\hline PZ1-c192 & 2439.2 & 34,391 & -1.7 & 32,400 & \pm & 400 & 12413 & 200 & 164 & \pm & 116 \\
\hline PZ1-c193 & 2415.2 & 34,727 & -1.0 & 32,500 & \pm & 400 & 12414 & 200 & 197 & \pm & 119 \\
\hline PZ1-c194 & 2330.2 & 35,917 & 0.8 & 32,500 & \pm & 400 & 12415 & 200 & 382 & \pm & 138 \\
\hline PZ1-c195 & 2327.6 & 35,954 & 1.8 & 33,900 & \pm & 400 & 12416 & 200 & 166 & \pm & 116 \\
\hline
\end{tabular}


Figure 2 shows the present $\Delta^{14} \mathrm{C}$ record for Lake Lisan corrected for variable reservoir ages (VR). The dashed line indicates the effect in $\Delta^{14} \mathrm{C}$ due to deviation from the $1250 \mathrm{yr}$ reservoir age. The present Lake Lisan $\Delta^{14} \mathrm{C}$ record agrees with the Lake Suigetsu record until $33 \mathrm{kyr}$ cal BP (the best fit with Lake Suigetsu data is obtained for Lisan reservoir ages of $2000 \mathrm{yr}$ between 20.9 and $25.2 \mathrm{kyr}$ cal BP, and $200 \mathrm{yr}$ between 32.1 and $36.0 \mathrm{kyr}$ cal BP). Structures at 18, 22, 31, and $32 \mathrm{kyr}$ cal BP are recognized in both records. Clearly, the structures at 18 and $22 \mathrm{kyr}$ cal BP are not represented well by the INTCAL98 curve. The close correspondence of the ${ }^{14} \mathrm{C}$ record of lakes Lisan and Suigetsu lends support to the varve counting of the latter in this interval. This means that the $\Delta^{14} \mathrm{C} \sim 0 \%$ observed in the Lake Lisan record at $32 \mathrm{kyr}$ cal BP is confirmed by the ${ }^{14} \mathrm{C}$ record of Lake Suigetsu, where no reservoir age effect plays a role in the ${ }^{14} \mathrm{C}$ data obtained from macrofossils. The new Lake Lisan record agrees with both the coral data, except for the coral data point at $30 \mathrm{kyr} c a l \mathrm{BP}$, and the Lake Lisan HS data (Haase-Schramm et al. 2004), except for the data point at $27 \mathrm{kyr}$ cal BP. At $34 \mathrm{kyr}$ cal BP, the Lake Suigetsu record shows a number of different $\Delta^{14} \mathrm{C}$ values (Figure 1) which may be indicative of a depositional hiatus. A 2-kyr hiatus would shift the data points to higher ages and higher corresponding $\Delta^{14} \mathrm{C}$ values (Figure 2) in agreement with Lake Lisan data.

While the ${ }^{14} \mathrm{C}$ record of Lake Lisan resembles the Lake Suigetsu record, both are different from the $\Delta^{14} \mathrm{C}$ values Bard (1998) modeled, based on the paleomagnetic compilation by Guyodo and Valent (1996) and the ${ }^{10} \mathrm{Be}$ production compilation by Frank et al. (1997). The present Lake Lisan record shows a maximum $\Delta^{14} \mathrm{C}$ of $\sim 450 \%$ at $25 \mathrm{kyr}$ cal BP and a minimum $\Delta{ }^{14} \mathrm{C}$ of $\sim 0 \%$ at $32 \mathrm{kyr}$ cal BP, while the model of Bard indicates steadily increasing $\Delta^{14} \mathrm{C}$ values toward a maximum $\Delta^{14} \mathrm{C}$ of $\sim 350 \%$ at $30 \mathrm{kyr}$ cal BP. Obviously, other parameters play a role in the ${ }^{14} \mathrm{C}$ record. The $\Delta^{14} \mathrm{C} \sim 0 \%$ at $32 \mathrm{kyr}$ cal BP deduced from lakes Lisan and Suigetsu suggests that the production of ${ }^{14} \mathrm{C}$ was similar at that time to the present day or the same combination of production with the global reservoir carbon exchange prevailed. This matter, which we regard as a fundamental observation, requires further study and modeling.

The similarity of the $\Delta^{14} \mathrm{C}$ records of lakes Lisan and Suigetsu lends support to the validity of both calendar chronologies up to $34 \mathrm{cal} \mathrm{ka} \mathrm{BP}$, but for ages $>25 \mathrm{kyr}$ cal BP, their $\Delta{ }^{14} \mathrm{C}$ values are clearly lower than the ${ }^{14} \mathrm{C}$ records of the Iceland Sea, the Cariaco Basin, and the Bahamas stalagmite. In the case of the stalagmite record, the reservoir age of $1450 \mathrm{yr}$, as derived for the interval of 11-16 kyr cal BP, was assumed for the whole age scale, which may not be justified. However, even zero reservoir age would not be sufficient to reduce the ${ }^{14} \mathrm{C}$ vales sufficiently for matching the other ${ }^{14} \mathrm{C}$ records. Only overestimation of the calendar age record can explain the difference with the Lake Lisan record. Reduction of the calendar age scale of the Iceland Sea and the Cariaco Basin - which were both determined from correlation with the GISP2 core - by 3\% and the stalagmite by $5-10 \%$, is sufficient to obtain a reasonable consensus with the present Lake Lisan record (Figure 3). Such a shift could indicate a wrong age assessment for the records of the Iceland Sea and the Cariaco Basin, which both depend on comparison with GISP2 data. In the case of the speleothems, the age assessment depends completely on the assumptions regarding the reservoir age and those related to the presence of initial Th and ${ }^{234} \mathrm{U} /{ }^{238} \mathrm{U}$ as well as the closed-system condition. The study of the behavior of the U-Th and ${ }^{14} \mathrm{C}$ system in the Lisan aragonite certainly indicated that unlike pristine corals, the initial Th factor can cause shifts in the U-Th calendar ages (Haase-Schramm et al. 2004).

\section{CONCLUSION}

We established a high-resolution ${ }^{14} \mathrm{C}$ record for Lake Lisan for the interval of $17-36 \mathrm{kyr}$ cal BP by analyses of authigenic aragonite and application of variable reservoir ages determined by aragoniteorganic debris pairs from the same stratigraphic horizons. The calendar ages of the aragonites were determined by U/Th (Haase-Schramm et al. 2004). 


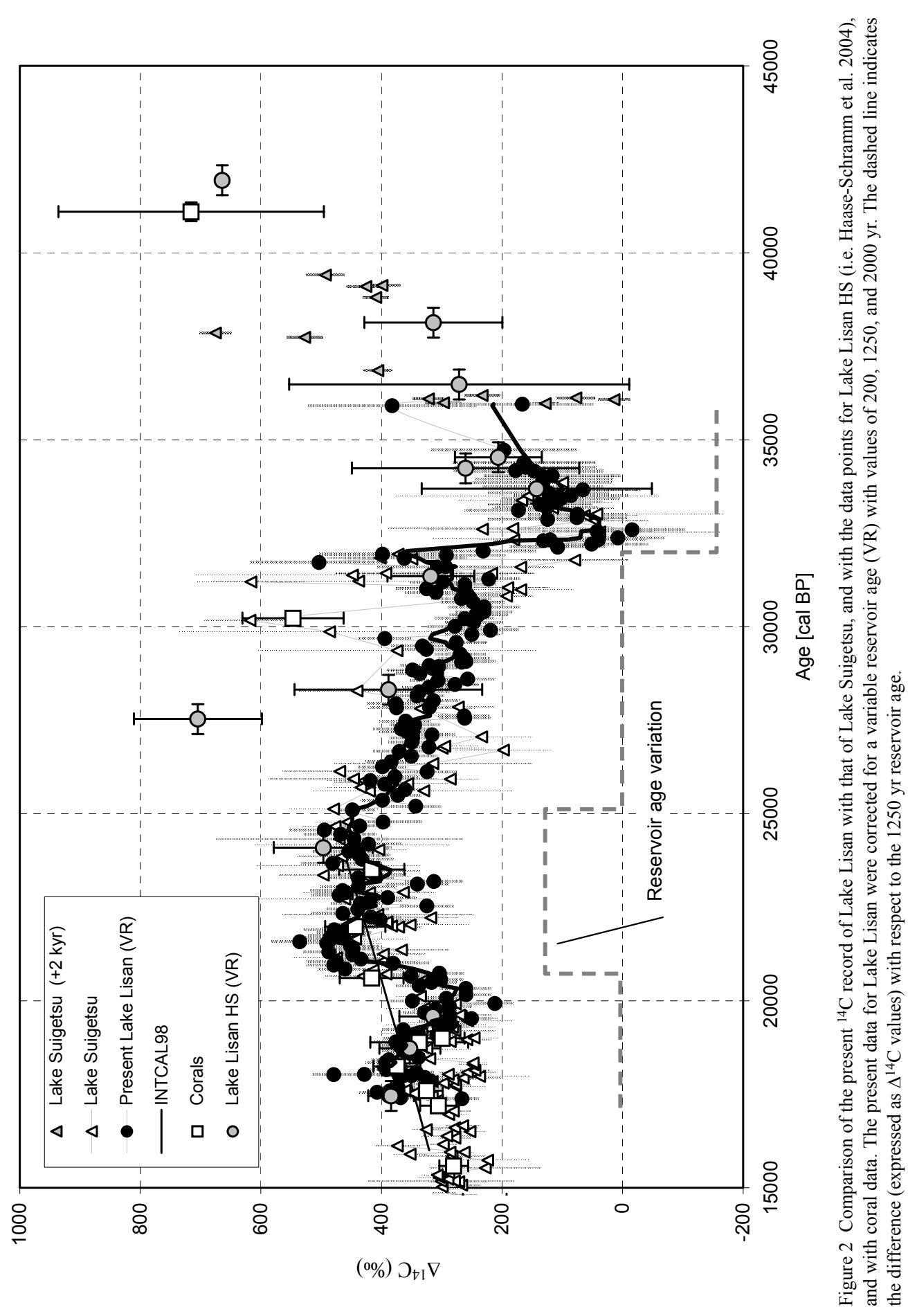




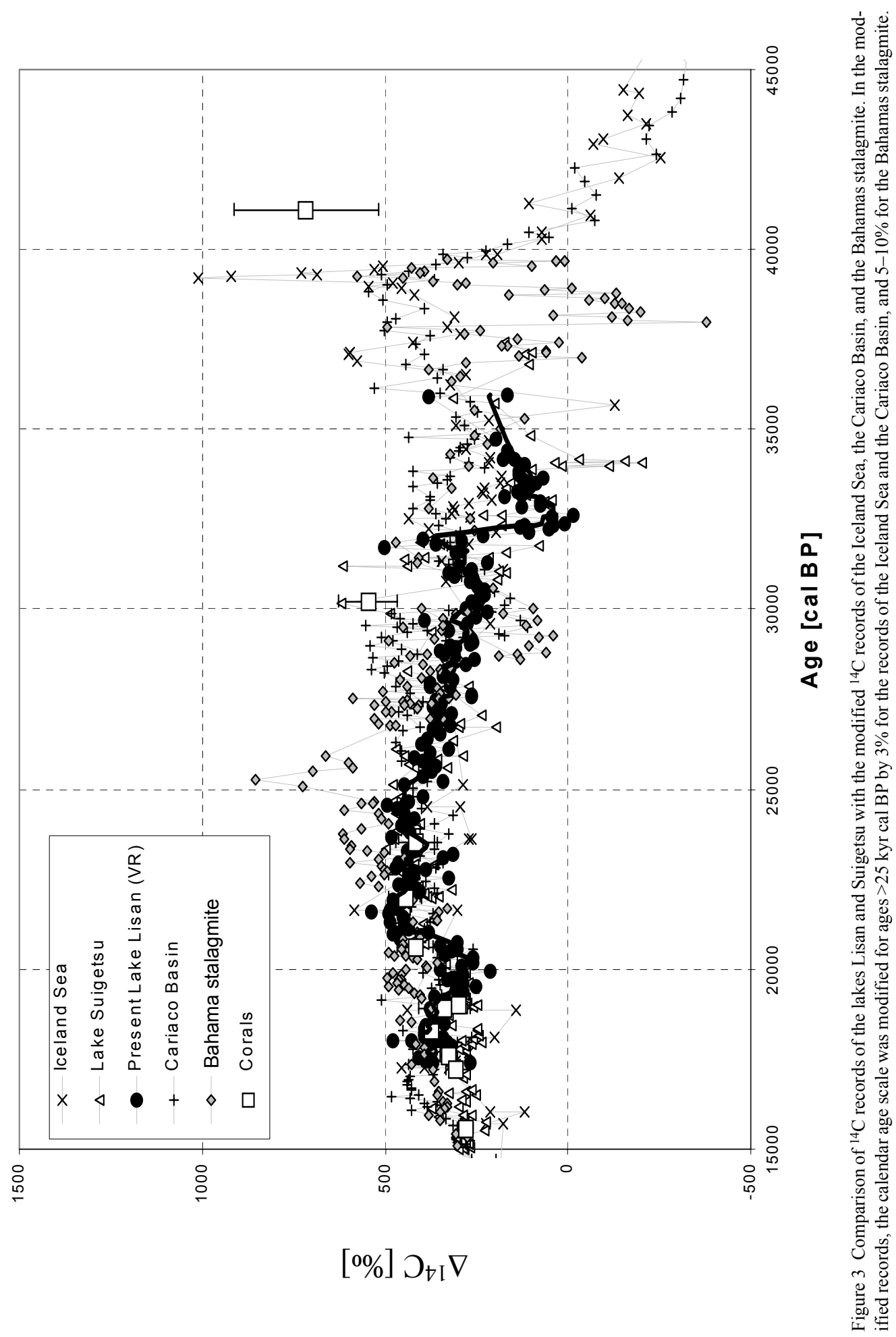


The present ${ }^{14} \mathrm{C}$ record of Lake Lisan resembles that from Lake Suigetsu. This observation supports both the validity of the varve counting used for the calendar age scale for Lake Suigetsu up to $33 \mathrm{kyr}$ cal BP, and the use of variable reservoir ages in the Lake Lisan record.

Both the Lisan and Suigetsu records converge to $\Delta^{14} \mathrm{C} \sim 0$ at $32 \mathrm{kyr}$ cal BP, suggesting an atmospheric production rate similar to present-day conditions or the same combination of production with the global reservoir carbon exchange.

The Lake Lisan and Suigetsu records do not show agreement with the modeled ${ }^{14} \mathrm{C}$ record, taking into account the effect of the geomagnetic field on the ${ }^{14} \mathrm{C}$ production. They also do not agree with the ${ }^{14} \mathrm{C}$ records of the Iceland Sea, Cariaco Basin, and the Bahamas stalagmite. However, a reasonable consensus with these other records is obtained by reducing the calendar age scale by $3 \%$ for the records of the Iceland Sea and the Cariaco Basin, and 5-10\% for the Bahamas stalagmite.

\section{ACKNOWLEDGEMENTS}

This work was supported by US-Israel Binational Science Foundation (USIBSF) Grant \#2000271 to MS and SLG. Eithan Shelef and Boaz Tatarski helped in field collection and the meticulous work of laminae separation.

\section{REFERENCES}

Bard E, Arnold M, Hamelin B, Tisnerat-Laborde N, Cabioch G. 1998. Radiocarbon calibration by means of mass spectrometric ${ }^{230} \mathrm{Th} /{ }^{234} \mathrm{U}$ and ${ }^{14} \mathrm{C}$ ages of corals: an updated database including samples from Barbados, Mururoa and Tahiti. Radiocarbon 40(3):1085-92.

Bard E. 1998. Geochemical and geophysical implications of the radiocarbon calibration. Geochimica et Cosmochimica Acta 62(12):2025-38.

Bartov Y, Goldstein SL, Stein M, Enzel Y. 2003. Catastrophic arid episodes in the Eastern Mediterranean linked with the North Atlantic Heinrich event. Geology 31:439-44.

Beck JW, Richards DA, Edwards RL, Silverman BW, Smart PL, Donahue DJ, Herrera-Osterheld S, Burr GS, Calsoyas L, Jull AJT, Biddulph D. 2001. Extremely large variations of atmospheric ${ }^{14} \mathrm{C}$ concentration during the last glacial period. Science 292:2453-7.

Begin ZB, Ehrlich A, Nathan Y. 1974. Lake Lisan-The precursor of the Dead Sea. Geological Society Survey Israel Bulletin 63:1-30.

Frank M, Schwarz B, Baumann S, Kubik PW, Suter M, Mangini A. 1997. A 200-kyr record of cosmogenic radionuclide production rate and geomagnetic field intensity from ${ }^{10} \mathrm{Be}$ in globally stacked deep-sea sediments. Earth and Planetary Science Letters 149:1219.

Guyodo Y, Valet J-P. 1996. Relative variations in geomagnetic intensity from sedimentary records: the past 200,000 years. Earth and Planetary Science Letters 143:23-36.

Haase-Schramm A, Goldstein SL, Stein M. 2004. U-Th dating of Lake Lisan aragonite (late Pleistocene Dead Sea) and implications for glacial East Mediterranean climate change. Geochimica et Cosmochimica Acta 68(5):985-1005.

Hughen K, Lehman S, Southon J, Overpeck J, Marchal
O, Herring C, Turnbull J. 2004. ${ }^{14} \mathrm{C}$ activity and global carbon cycle changes over the past 50,000 years. Science 303:202-7.

Kaufman A. 1971. U-series dating of Dead Sea Basin carbonates. Geochimica et Cosmochimica Acta 35: 1269-81.

Kitagawa H, van der Plicht J. 2000. A 40,000-yr varve chronology from Lake Suigetsu, Japan: extension of the ${ }^{14} \mathrm{C}$ calibration curve. Radiocarbon 42(3):369-80.

Schramm A, Stein M, Goldstein SL. 2000. Calibration of the ${ }^{14} \mathrm{C}$ timescale to $>40$ ka by ${ }^{234} \mathrm{U}-{ }^{230} \mathrm{Th}$ dating of Lake Lisan sediments (Last Glacial Dead Sea). Earth and Planetary Science Letters 175:27-40.

Stein M, Starinsky A, Katz A, Goldstein SL, Machlus M, Schramm A. 1997. Strontium isotopic, chemical and sedimentological evidence for the evolution of Lake Lisan and the Dead Sea. Geochimica et Cosmochimica Acta 61(18):3975-92.

Stein M, Migowski C, Bookman R, Lazar B. 2004. Temporal changes in radiocarbon reservoir age in the Dead Sea-Lake Lisan system. Radiocarbon, these proceedings.

Stuiver M, Reimer PJ, Bard E, Warren Beck J, Burr GS, Hughen KA, Kromer B, McCormac G, van der Plicht J, Spurk M. 1998. INTCAL98 radiocarbon age calibration, 24,000-0 cal BP. Radiocarbon 40(3):104183.

van der Borg K, Alderliesten C, de Jong AFM, van den Brink A, de Haas AP, Kersemaekers HJH, Raaymakers JEM. 1997. Precision and mass fractionation in ${ }^{14} \mathrm{C}$ analysis with AMS. Nuclear Instruments and Methods in Physics Research B 123:97-101.

Voelker AHL, Grootes PM, Nadeau M-J, Sarntheim M. 2000. Radiocarbon levels in the Iceland Sea from 25 $53 \mathrm{kyr}$ and their link to the earth's magnetic field intensity. Radiocarbon 42(3):437-52. 